\title{
Review
}

\section{Recent advances in peptide nucleic acid for cancer bionanotechnology}

\author{
Jun-chen WU ${ }^{1, *}$, Qing-chun MENG ${ }^{1,2}$, Hong-mei REN ${ }^{1,2}$, Hong-tao WANG ${ }^{2}$, Jie WU ${ }^{3}$, Qi WANG ${ }^{3, *}$ \\ ${ }^{1}$ Key Laboratory for Advanced Materials and Institute of Fine Chemicals, School of Chemistry and Molecular Engineering, East China \\ University of Science and Technology, Shanghai 200237, China; ${ }^{2}$ Research Institute of Exploration and Development, PetroChina \\ Huabei Oilfield Company, Renqiu 062500, China; ${ }^{3}$ College of Public Health, Nantong University, Nantong 226019, China
}

\begin{abstract}
Peptide nucleic acid (PNA) is an oligomer, in which the phosphate backbone has been replaced by a pseudopeptide backbone that is meant to mimic DNA. Peptide nucleic acids are of the utmost importance in the biomedical field because of their ability to hybridize with neutral nucleic acids and their special chemical and biological properties. In recent years, PNAs have emerged in nanobiotechnology for cancer diagnosis and therapy due to their high affinity and sequence selectivity toward corresponding DNA and RNA. In this review, we summarize the recent progresses that have been made in cancer detection and therapy with PNA biotechnology. In addition, we emphasize nanoparticle PNA-based strategies for the efficient delivery of drugs in anticancer therapies.
\end{abstract}

Keywords: peptide nucleic acids; bionanotechnology; cancer diagnostics and therapy

Acta Pharmacologica Sinica (2017) 38: 798-805; doi: 10.1038/aps.2017.33; published online 17 Apr 2017

\section{Introduction}

Peptide nucleic acid (PNA) was originally invented in 1991 by Peter E NIELSEN (University of Copenhagen), Michael EGHOLM (University of Copenhagen), Rolf H BERG (Risø National Laboratory), and Ole BUCHARDT (University of Copenhagen) to act as a sequence-specific DNA binding reagent ${ }^{[1]}$. PNAs play an important role as therapeutic agents, anticancer agents, molecular tools and biosensor probes, as well as in nanotechnology. PNA bionanotechnology, which includes nanoparticles, nanodevices, and nanoscale phenomena within the discipline of nanotechnology, affords a new approach for scientists to create new functional materials for cancer detection and therapy. PNAs have a distinct N-(2-aminoethyl)glycine backbone. Purines and pyrimidines are then connected to the backbone by methylene carbonyl linkages ${ }^{[2]}$. It is easy to hybridize DNA/PNA sequences because the structure of PNA is similar to natural nucleic acid (Figure 1). Specific PNA oligomers have been used as anticancer therapeutics to detect tumor cells or deliver small molecule drugs targeting nucleic acids ${ }^{[3,4]}$. However, all reported methodologies require labeling or conjugating steps, which are time-consuming and inefficient. Nanotechnology provides direct, simple, rapid

\footnotetext{
${ }^{*}$ To whom correspondence should be addressed.

E-mail jcwu@ecust.edu.cn (Jun-chen WU); wangqi@ntu.edu.cn (Qi WANG)

Received 2016-11-02 Accepted 2017-02-04
}

and label-free methods for PNA application. This technical approach provides an extremely attractive solution for cancer diagnosis and therapy.

Nanoparticles usually act as a bridge between bulky materials and molecular structures. Incorporating PNAs with nanoparticles enables the complexes to possess the properties of both nanoparticles and PNAs. For example, nanoparticles often possess optical properties. Surface-coating fluorescent nanoparticles could be used to image tumor tissues or RNA molecules in living cells ${ }^{[5]}$. Some nanoparticle-PNA complexes have high surface-to-volume ratios, which greatly enhance the detection limit of biosensors. As PNA has an uncharged backbone, PNA/DNA duplexes are more stable than the DNA/DNA equivalents. Therefore, single-base mismatches of PNA/DNA duplexes have a better destabilizing effect. The neutral amide backbone of PNAs has a significant advantage in that it can hybridize with DNA under low salt conditions. This indicates that there is no need to counteract the interstrand repulsion between the two negatively charged nucleic acids with positive ions. PNA oligomers can not only effectively bind to complementary DNA/RNA but also have extensive storage temperature and $\mathrm{pH}$ ranges ${ }^{[6]}$. The unnatural backbone of PNAs makes them resistant to both nucleases and proteases $^{[7]}$. Based on the excellent chemical and biological stability of PNAs, there is a growing interest in PNAs as gene therapy drugs, diagnostic reagents and molecular beacons ${ }^{[8-10]}$. In this review, we summarize the current research on PNA 


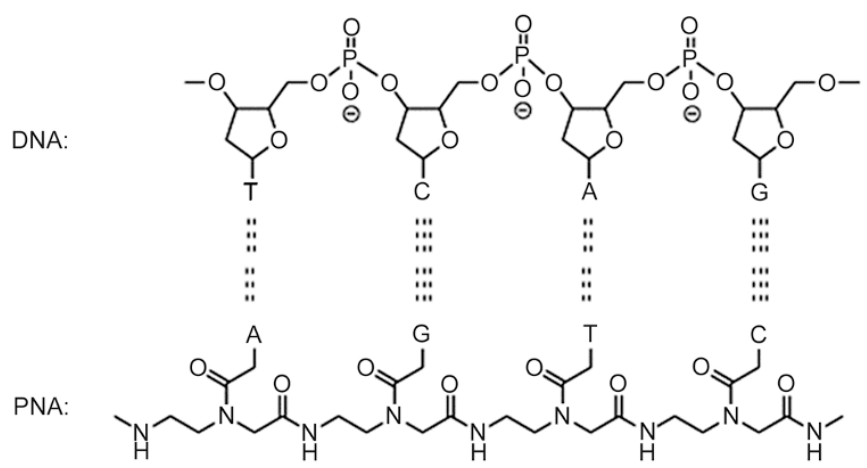

Figure 1. PNAs hybridize to complementary DNA by hydrogen bonding. $A=$ adenine,$G=$ guanine,$C=$ cytosine and $T=$ thymine.

bionanotechnology in cancer treatment and its limitations. We focus on the use of nanoparticle-based delivery systems, PNA anticancer drugs for tumor therapy, and PNA probes for cancer diagnosis.

\section{Synthesis of PNA}

In recent years, PNA oligomers have been used as antibacterial and antisense drugs, as well as for nucleic acid diagnosis and molecular biology. With the excellent binding ability of PNA, it is unnecessary to design long PNA oligomers for these applications. However, specificity is the main concern in designing long PNA oligomers. Unlike the phosphodiester backbone of nucleic acids, a PNA has an achiral backbone of $\mathrm{N}$-(2-aminoethyl)glycine, which connects to the nucleotide bases. This unique achiral structure enables PNAs to be easily synthesized without considering stereoselectivity.

The synthesis of PNA oligomers can be carried out easily by a standard, solid-phase protocol using Fmoc-protected PNA monomers ${ }^{[1-13]}$. Typically, the PNA oligomers are deprotected and cleaved off of the resin using 4-trifluoromethylsalicylic acid (TFMSA)/trifluoroacetic acid (TFA) and then purified by reverse-phase high-performance liquid chromatography (HPLC). PNA oligomers can be characterized by matrixassisted laser desorption/ionization time-of-flight (MALDITOF) mass spectrometry. PNA-peptide conjugates can be synthesized by continuous synthesis using standard peptide conjugation techniques, such as maleimide cysteine coupling or thioester condensation. Inspired by the synthesis of PNAs, a large number of PNA analogs ${ }^{[14]}$, such as a variety of nonnatural nucleobases, have been synthesized ${ }^{[15-18]}$ (Figure 2). The amino groups of the PNA monomers are usually protected by Fmoc (9-fluorenylmethoxycarbonyl group) or Bhoc (benzhydryloxycarbonyl group) $^{[12]}$. Originally, PNA monomers were connected on a Merrifield resin (methylbenzhydrylamine) in a fritted vial. After capping the unreacted groups in resin, the Fmoc group was deprotected by adding piperidine solution (20\% piperidine in dimethylformamide). The following monomers of PNA were connected to the resin one after another by reacting with the corresponding coupling reagents. At the end of the synthesis, the Fmoc group was removed by a piperidine solution. The Bhoc group, which protects the A, C, G, T monomers, was removed by trifluoroacetic acid (Figure 3 ).

\section{Advantages of PNA}

Thermal stability of PNA and its hybrid complexes

PNA oligomers have several important advantages for biological applications. As compared with DNA/DNA and DNA/RNA duplexes, PNA/RNA and PNA/DNA duplexes have excellent thermal stability ${ }^{[19]}$. The neutral PNA backbone

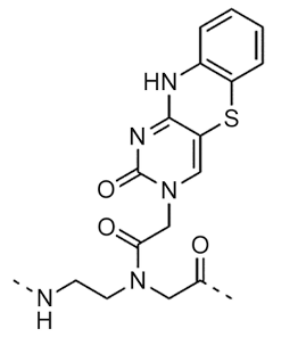

tC

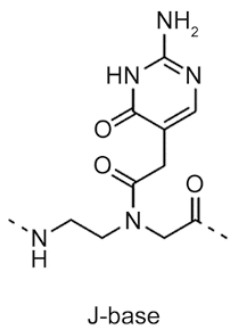<smiles>CNCCN(CC(C)=O)C(=O)Cc1cc2ccc(Cl)nc2[nH]c1=O</smiles>

7Cl-bT

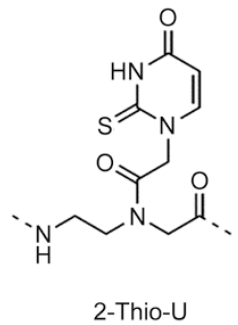<smiles>CNCCN(CC(C)=O)C(=O)Cn1cnc2c(N)nc(N)nc21</smiles>

DAP<smiles>CNCCN(CC(C)=O)C(=O)CCNc1ccc(=O)[nH]n1</smiles>

Figure 2. Fmoc/Bhoc-protected PNA monomers. 


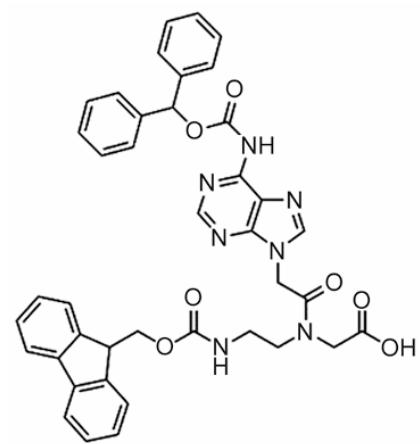

Fmoc-PNA-A(Bhoc)-OH

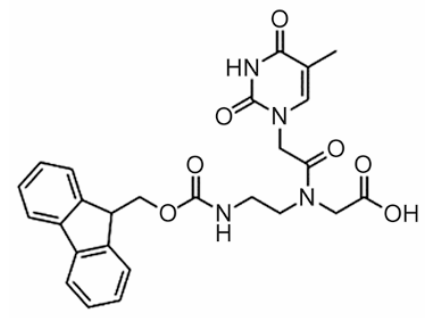

Fmoc-PNA-T-OH

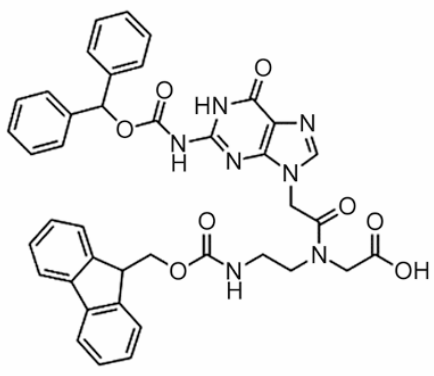

Bhoc:<smiles>[Y]C(=O)OC(c1ccccc1)c1ccccc1</smiles>

Fmoc-PNA-G(Bhoc)-OH

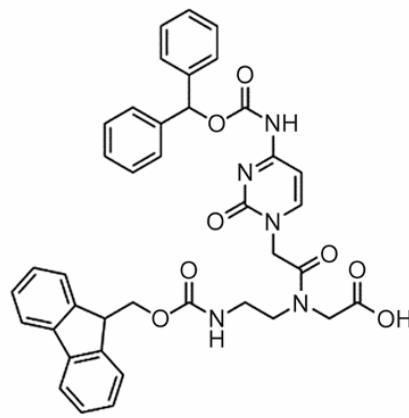

Fmoc:<smiles>CC(=O)OCC1c2ccccc2-c2ccccc21</smiles>

Figure 3. Chemical structures of the unnatural nucleobases used in PNA oligomers.

lacks a repulsion charge when binding with DNA/RNA, making the complexes more stable. As reported by Shakeel et al, a 15-mer PNA/DNA or PNA/RNA duplex has a $70^{\circ} \mathrm{C}$ melting temperature, whereas the corresponding DNA/DNA or DNA/RNA duplexes are approximately $55^{\circ} \mathrm{C}$. Generally, in $100 \mathrm{mmol} / \mathrm{L} \mathrm{NaCl}$ solution, the melting temperature of PNA/ DNA duplexes is $1^{\circ} \mathrm{C}$ higher (per base pair), as compared with the melting temperature of the corresponding DNA/RNA duplexes. For a 15-mer PNA/DNA or PNA/RNA duplex, the melting temperature values are approximately $20^{\circ} \mathrm{C}$ higher than the natural nucleic acid duplexes ${ }^{[7]}$.

\section{Stronger binding ability in high ionic strength}

The neutral backbone enables PNAs to form stable PNA/DNA duplexes at high salt concentrations. The melting temperatures of the duplexes in high salt concentration are higher than the DNA/DNA duplexes ${ }^{[20]}$. At low ionic strength, at certain temperatures, PNAs have a stronger ability to bind complementary DNA/RNA sequences than DNA hybridization. In contrast to $\mathrm{Mg}^{2+}$ inhibition of DNA/DNA duplex formation, hybridization of a PNA can be carried out in the absence of $\mathrm{Mg}^{2+}$. Therefore, the appropriate ionic strength can eliminate the influence of DNA/RNA in the sample.

\section{Greater interaction specificity}

Another advantage is the greater specificity of PNA when interacting with complementary PNA/RNA ${ }^{[21]}$. A PNA/ DNA or PNA/RNA base pair mismatch usually is much more destabilizing than a mismatch in a neutral nucleic acid duplex. On average, a single mismatch in PNA/DNA or PNA/RNA 15 -mers decreases the melting temperature by $15^{\circ} \mathrm{C}$. Additionally, a single mismatch of the DNA/DNA duplexes lowers the melting temperature by $11^{\circ} \mathrm{C}$.

\section{Strong strand invasion ability}

PNA oligomers have a strong strand invasion ability. PNA oligomers that contain cytosine and thymine usually bind with nucleic acids in a 2/1 (PNA/DNA) manner and form PNA/ DNA/PNA triplexes ${ }^{[22,23]}$. The triplex is formed by first assembling a PNA/DNA duplex. Then, the second PNA oligomer is inserted into the major groove of the PNA/DNA double helix. Because the triplexes are very stable (melting temperature $>70^{\circ} \mathrm{C}$ for a 10 -mer complex), PNAs can even invade the strands of neutral nucleic acid duplexes by displacing one of the DNA strands and forming a D loop in the double-stranded DNA. This characteristic has been applied in gene expression analysis.

\section{Resistance to nucleases and proteases}

The different skeletons of PNAs (as comparing with DNA/ RNA) determine their peptide backbone and are not easily degraded by nucleases or proteases ${ }^{[6]}$.

\section{PNA bionanotechnology for cancer therapy} PNA as anticancer drug carriers

PNAs designed as cancer drugs are valuable for cell-specific 
nucleic acid delivery because they can strongly bind to specific nucleic acids (eg, microRNA). In some diseases, microRNAs, for example miR-21, are key regulators of oncogenic processes and are usually overexpressed ${ }^{[24-26]}$. MicroRNAs are endogenous short RNAs that have important cellular functions ${ }^{[27-30]}$ and they are ideal diagnostic and therapeutic targets in cancer therapy ${ }^{[31,32]}$.

Lee et al developed a miRNA-responsive theranostic platform in the treatment of cancer ${ }^{[33]}$. The microRNA-responsive drug is composed of a photosensitizer, chlorin e6 (Ce6), which is connected to the PNA sequence and the dextran-coated reduced graphene oxide nanocolloid (Dex-RGON). The synthesized PNA has a complementary sequence to cancerspecific miR-21, which can selectively hybridize to cancer cells. Upon binding to cancer cells, Ce6-PNA is released from DexRGON, and the fluorescence of Ce6 is restored. Through nearinfrared irradiation, Ce6 acts as a photosensitizer and can kill cancer cells.

To increase the selectivity and transfection efficiency of therapeutic nucleic acids in tumor cells, Zhang et al developed a dual receptor-targeted quantum dots (QDs) gene carrier ${ }^{[34]}$. The carrier is composed of four segments: CdSe/ZnS QDs, biotinylated anti-survivin PNAs (anti-survivin antisense oligonucleotides: 5'-(biotin-SS-)-CCCAGCCTTCCAGCTCCTTG-3'), streptavidin and two targeting peptides, GE11 and c(RGDfK). The target PNA sequence can selectively bind to epidermal growth factor receptors (EGFRs) and integrin $a v \beta 3$ receptors, which increase the cellular uptake of QD carriers. The gene carrier can effectively increase the efficiency of gene silencing and shows the potential for great application in cancer therapy. Furthermore, they investigated a quantum dot probe for detecting the down-regulation of folate receptor $(\mathrm{hFR})$ in breast cancer cells. The probe is composed of a biotinylated antisense oligonucleotide (AS-ODN, a 21-mer single-strand DNA molecule), a biotinylated p160 peptide (VPWMEPAYQRFL) and a streptavidin-coated core-shell CdSe/ZnS QD. The probe could specifically target MCF-7 human breast cancer cells and real-time track AS-ODN cellular delivery. ASODNs can bind to hFR and inhibit its expression. Thus, with the binding of $\mathrm{hFR}$, the probe can be used to track gene function in anticancer therapy ${ }^{[35]}$.

\section{PNA as cancer drugs}

The development of chemical approaches for controlling gene expression will facilitate investigations of new gene therapy drugs for cancer therapy ${ }^{[36]}$. PNAs are perfect candidates in the design of gene therapeutic drugs due to their strong strand invasion ability and both chemical and biological stabilities $^{[37-39]}$. Two strategies have been involved in using PNAs as therapeutic drugs, namely, antisense and anti-gene therapies.

Antisense oligonucleotides have been widely used to block the expression of genes or alter splicing ${ }^{[40]}$. The antisense effect of PNA is normally due to the steric blocking of RNA transport and translation into the cytoplasm. PNAs have exceptionally high affinity to complementary sequences and possess an uncharged backbone; thus, it is naturally difficult for them to interact with many cellular proteins that bind anionic macromolecules ${ }^{[41]}$. In vitro translation experiments with rabbit reticulocyte lysates display that PNAs are capable of inhibiting translation by overlapping the AUG start codon at the targets and forming duplex and triplex complexes. In the mRNA coding region, PNAs are able to bind the translation machinery by forming triplexes. Unlike duplex-forming PNAs, the formation of the triplex requires a target containing 10 to 15 homopurine bases. Triplex-forming PNAs have the ability to inhibit translation at initiation codon targets.

PNAs have been used to image the expression of an endogenous gene in brain cancer and to act as a targeted PNA radiopharmaceutical ${ }^{[42]}$. Suzuki et al designed two PNAs, which were antisense to either rat caveolin-1- (CAV) mRNA or rat glial fibrillary acidic protein (GFAP) mRNA. These two PNAs contain an amino-ended diethylenetriaminepentaacetic acid and a carboxyl-terminal-N-biotinyl lysine moiety. The aminoterminal diethylenetriaminepentaacetic acid was designed to chelate radioactive ${ }^{111} \mathrm{In}$, and the carboxyl-terminal-biotinyl lysine residue was used to connect with the delivery system (Figure 4). The carboxyl-terminal-biotinyl lysine residue is composed of streptavidin (SA) and a murine OX26 monoclonal antibody, which is connected by a thiol-ether linker. The control group PNAs were designed without SA-OX26. By attaching the PNA antisense agent, the antisense radiopharmaceutical is able to penetrate the membrane barriers and access the target mRNA. The PNA-modified radiopharmaceutical can then be used to image endogenous gene expression. Another strategy is the use of PNAs as anti-gene therapy drugs. The unmatched ability of PNAs to invade duplex DNA suggests that they can bind to chromosomal targets and act as anti-gene agents. For this purpose, PNAs were synthesized to recognize or hybridize to specific sequences in a particular gene. By forming a triplex or a strand invasion complex with
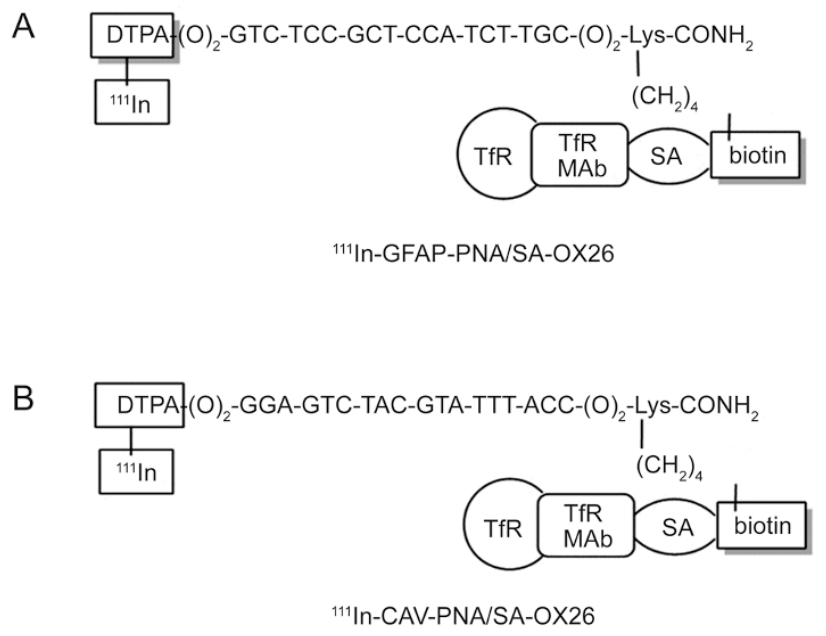

Figure 4. (A) The structure of an 18-mer PNA which is antisense to rat GFAP mRNA. (B) The structure of an 18-mer PNA, which is antisense to rat CAV mRNA. TfR represents rat transferrin receptor; SA represents streptavidin. 
DNA, PNAs are capable of arresting transcriptional processes. These complexes can work as anti-gene agents by blocking the stable functioning of RNA polymerase through the formation of a structural hindrance. In vitro studies show that the transcription processes of both prokaryotic and eukaryotic RNA polymerases can be affected via these complexes. Moreover, PNAs can also affect polymerase progression and transcription elongation by targeting the promoter regions or further downstream from the promoter region.

PNAs are further used in gene editing technology. McNeer et al found that PNA/DNA-containing nanoparticles are more effective than DNA-containing nanoparticles to produce in vivo gene editing in human hematopoietic cells ${ }^{[8]}$. Instead of gene knock-in, this non-toxic, virus-free poly(lactic-co-glycolic acid) (PLGA) nanoparticle can be easily reengineered to target various genes, cells and mediate site-specific modification in human hematopoietic stem and progenitor cells (HSPCs) in vitro. This high efficiency and low toxicity gene editing technology can be applied in the treatment of cancer, which involves multiple organs and target cells in vivo.

PNAs as antisense and anti-gene therapy drugs open the door for anticancer drug design. However, unlike nucleic acid oligonucleotides, it is difficult for the N-(2-aminoethyl) glycine backbone of PNAs to enter cells by endocytosis ${ }^{[43]}$. Many novel strategies have been explored to increase the ability of PNAs to penetrate the cell. One strategy is to graft the positively charged residues to PNA molecules. For example, lysine and arginine have been introduced into PNA sequences to make them more accessible from the cell membrane. Short peptides or other ligands, such as antibodies and steroids, are involved in the construction of PNAs to increase the efficiency of delivery ${ }^{[44,45]}$.

\section{PNA bionanotechnology for cancer detection}

PNAs were originally designed for the recognition of doublestranded DNA. However, their extraordinary thermal stability and synthesis technology enable their potential use as excellent probes for cancer detection.

\section{PNA-silicon nanowire biosensor for detection of miRNAs}

MicroRNAs (miRNAs) are small non-coding RNA molecules found in animals and plants. MicroRNAs play an important role in gene regulation. Because many miRNAs are overexpressed in cancer diseases ${ }^{[46]}$, the detection of miRNAs is crucial in cancer diagnosis. PNA has a neutral backbone, which increases its hybridization ability with miRNA. Using PNAfunctionalized silicon nanowires (SiNWs), single base differences in miRNA can be discriminated. Thus, specific miRNAs can be identified in the total RNA extracted from cancer cells. Furthermore, SiNWs have a high surface-to-volume ratio, which enhances the sensitivity of the detection.

Zhang et al reported a label-free PNA-SiNWs biosensor for the ultrasensitive detection of miRNA. The biosensor is composed of an SiNW array and a PNA molecule. The PNA is covalently immobilized on the electrically addressable SiNW surface and serves as a receptor to recognize miRNAs. The biosensor can sensitively bind to miRNAs without labeling. The excellent sensitivity of the biosensor is suitable for the early detection of miRNAs in cancer diagnostics ${ }^{[47]}$.

\section{PNA as a detector for nucleic acid single base detection}

The detection of specific single bases in nucleic acid sequences is of great importance in the diagnosis of inherited diseases and genetic disorder-induced cancers. Short PNA oligomers have excellent thermal stabilities upon binding to nucleic acids. Single base mismatches will affect the thermal stability of the effectively formed duplex. Thus, PNA oligomers have potential applications in the detection of single, specific nucleic acid bases. Ficht et al reported that several labeled PNA probes can be used to detect specific, single DNA nucleotides. Using native chemical ligation, two adjacent PNA probes were hybridized together, which then served as an indicator for the target $\mathrm{DNA}^{[48-51]}$.

\section{PNA as a biosensor for the detection of nucleic acids}

PNAs have attracted increasing interest as biosensors for the detection of nucleic acids. PNA biosensors display real-time spatial and temporal resolution in the detection of nucleic acids. In living cells, they can distinguish bound DNA/RNA from unbound with a high signal-to-background ratio. Currently, as compared with traditional detection methods, PNA probes exhibit significant advantages in nucleic acid detection for cancer diagnosis ${ }^{[52]}$. Ostromohov et al developed a novel assay that can rapidly detect nucleic acids with high sensitivity. The detection assay is composed of isotachophoresis (ITP) and PNA probes. PNA probes have low electrophoretic mobility, which cannot be identified under ITP unless bound to their complementary sequence. In this way, they can easily identify nucleic acid sequences via ITP focusing ${ }^{[33]}$.

PNA oligonucleotides with unnatural backbones have been used as molecular beacons (MBs) for the detection of nucleic acids due to their excellent sensitivity and selectivity. PNA MBs are composed of a fluorescent dye and a quencher molecule at each terminus. They have stable stem-loop structures, which are composed of a fluorescent reporter and a quencher. By transferring energy to a proximate quencher, the fluorescence of the reporter dye can be effectively quenched in solution $^{[54]}$. The mechanism is achieved first by hybridizing with the nucleic acid sequence. Then, the stem-loop structure of the PNA is destroyed by hybridizing with DNA/RNA, which separates the fluorescent dye and the quencher. PNA beacons have unique, thermodynamically stable structure, efficient intrinsic signal switching, diverse fluorophore-quencher pairs, and good sensitivity and selectivity, making them excellent candidates for the detection of nucleic acids ${ }^{[55-59]}$.

PNA MBs usually bind to double-stranded DNA in the major groove via four different hybridizing modes (Figure 5). Among these four binding modes, double duplex invasion requires a DNA sequence with at least 50\% adenine-thymine content, whereas the other three modes require a homopyrimidine or homopurine DNA sequence ${ }^{[60]}$. A well-designed PNA $\mathrm{MB}$ can be used as a powerful tool for cancer cell imaging. 


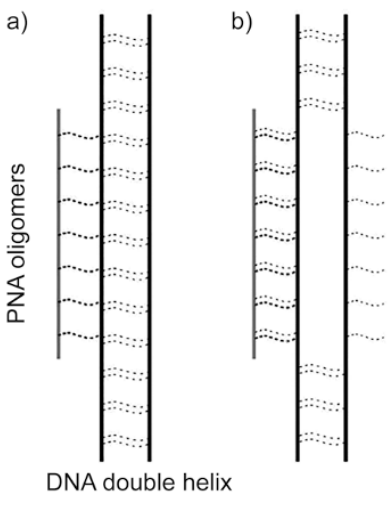

Figure 5. The scheme shows that PNA has 4 different ways to hybridize duplex DNA. a) Conventionally-formed triplex; b) Triplex invasion complexes; c) Double duplex invasion complexes; and d) Duplex invasion complexes.

Kam et al reported a PNA molecular beacon that served as a diagnostic probe for in vitro and ex vivo detection of colorectal cancer (CRC). The molecular beacon was composed of a colon cancer-associated transcript 1 (CCAT1) specific PNA, a thiazole orange (TO) fluorescent dye and a cell-penetrating peptide. They observed the in situ hybridization of a PNA beacon containing CCAT1 with human CRC specimens. This PNA $\mathrm{MB}$ is a powerful diagnostic tool for the detection of $C R C^{[61]}$.

Because PNA oligomers have good stability and specific hybridization with nucleic acids, PNA MBs are ideal for nucleic acid detection ${ }^{[62,63]}$. Ortiz et al reported a PNADNA MB that was composed of the fluorescent dye 7-amino4-methylcoumarin-3-acetic acid and the fluorescent quencher p-(dimethylaminophenylazo) benzoic acid (Figure 6). Upon binding to a complementary DNA sequence, the probe underwent fluorescence enhancement.

PNA MBs can also be used to detect quadruplex DNA. Datta et al reported a PNA MB that could invade quadruplex structures of the thrombin binding aptamer (TBA) and form stable complexes ${ }^{[64]}$.

\section{PNA probe for microarray}

PNAs have been used in microarrays ${ }^{[65]}$. Unlike DNA microarrays, PNA microarrays exhibit excellent sensitivity, selectivity and stability under various conditions. Due to their unique properties, PNA chips have better accuracy, good reproducibility and lengthier storage time. PNA microarrays have been used for the study of pathogen identification and functional genomics. The capability of PNA microarrays largely depends on the properties for attaching PNA molecules to the surface.

Brandt et al used PNA microarrays to hybridize unlabeled DNA samples. PNAs were first complexed with gold electrodes on the chip surface. DNAs were then added to the microarray. After hybridization with PNAs, the complexes were further bound by adding the intercalating dye. These hybridization events were monitored by electrochemical methods (Figure 7) ${ }^{[66,67]}$.

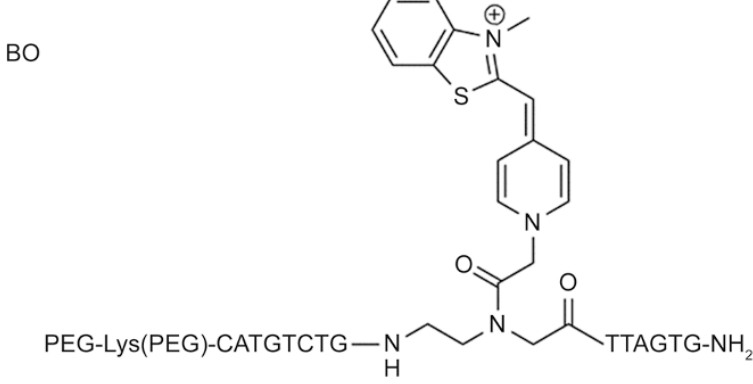

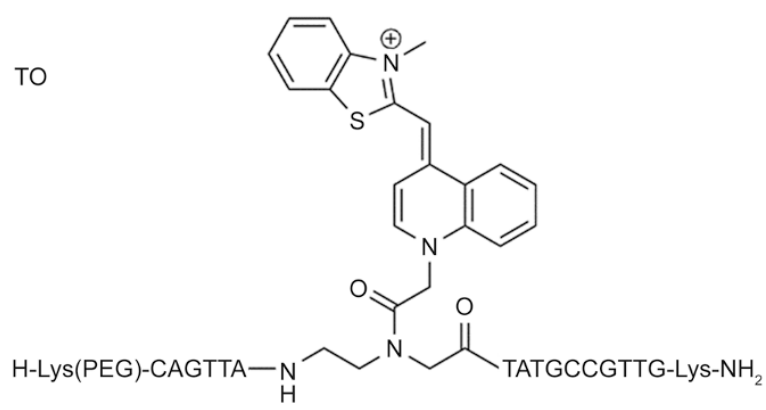

Figure 6. Chemical structures of the PNA intercalating dyes (BO, TO).

\section{Other PNA nanotechnologies Using PNA in southern hybridization}

Using fragments of defined length derived from restriction enzyme digestion, southern hybridization is usually used to analyze genetic loci. Southern hybridization is very useful because it can provide precise size and sequence information of nucleic acids for cancer diagnosis. Pre-gel hybridization of PNA reduces the cumbersome post separation, probing and washing steps in the process of southern hybridization. PerryO'Keefe et al prepared denatured DNA to hybridize with fluorescently labeled PNA oligomers at low ionic strength. By hybridizing with PNA, single-stranded DNA fragments exhibited higher electrophoretic mobility. After separation, the PNA/DNA hybrids were detected using standard chemiluminescent techniques. PNA is ideal for the detection and separation of specific DNA sequences because it has the ability to bind with DNA at low ionic strength. This technology enables cancer diagnosis to be faster and more accurate than conventional southern hybridization techniques ${ }^{[68]}$.

\section{PNA for nucleic acid purification}

PNA can hybridize and purify nucleic acids in low-salt concentrations that destabilize native nucleic acid structures. PNAs have high binding affinities, which can be used to purify specific nucleic acids. This technology has potential application in cancer research. Orum et al designed an oligo-histidine PNA with six histidine residues to purify a target nucleic acid. The designed $6 \times$ His-PNA could strongly bind to $\mathrm{Ni}(\mathrm{II})$ ions. By using a Ni(II)-NTA (nitrilotriacetic acid) resin, the $6 \times$ HisPNA/DNA complex could be purified by metal ion affinity 


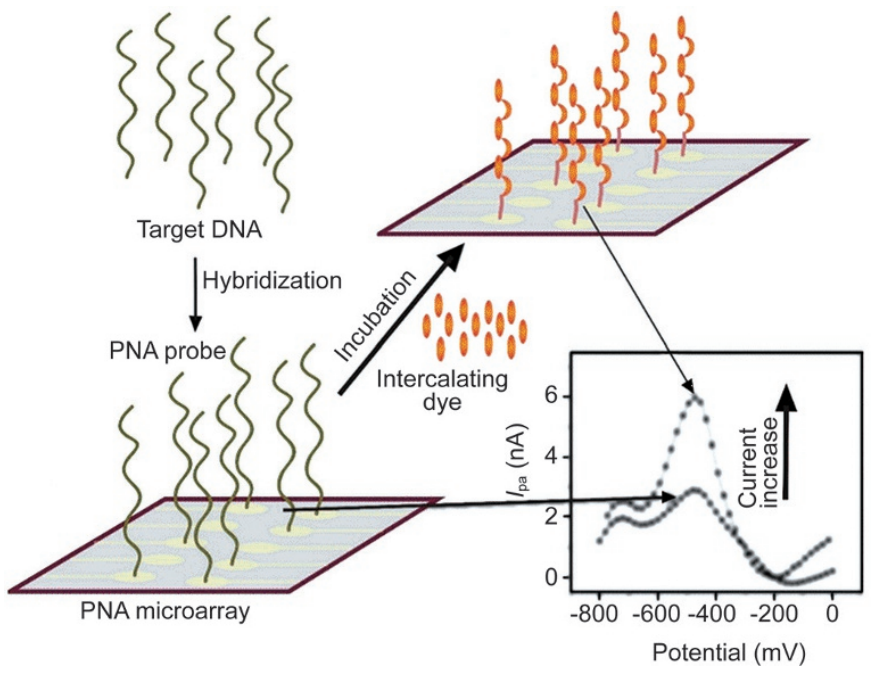

Figure 7. PNA microarray for the detection of target DNA on gold electrodes.

chromatography. They further demonstrated that large RNAs (2224 nucleotides) can be captured with high efficiency using multiple $6 \times$ His-PNA probes ${ }^{[6]]}$.

\section{Conclusions}

Peptide nucleic acids are of the utmost importance in the biomedical field because of their ability to hybridize with neutral nucleic acids and their special chemical and biological properties, which ensure that PNA oligomers are ideal candidates for application in the fields of cancer diagnosis and therapy. With future research efforts, PNA bionanotechnology will be able to develop an even wider field of application.

\section{Acknowledgements}

We thank the National Natural Science Foundation of China (21572057, 91529101 and 21341001) for financial support and the Innovation and Entrepreneurship Training Program of Jiangsu Province (2014105, 201510304036z) for financial support.

\section{References}

1 Nielsen PE, Egholm M, Berg RH, Buchardt O. Sequence-selective recognition of DNA by strand displacement with a thymine-substituted polyamide. Science 1991; 254: 1497-500.

2 Nielsen PE, Egholm M. An introduction to peptide nucleic acid. Curr Issues Mol Biol 1999; 1: 89-104.

3 Monroig PC, Chen L, Zhang S, Calin GA. Small molecule compounds targeting miRNAs for cancer therapy. Adv Drug Deliv Rev 2015; 81: 104-16.

4 Sheng J, Gan J, Huang Z. Structure-based DNA-targeting strategies with small molecule ligands for drug discovery. Med Res Rev 2013; 33: 1119-73.

5 Suzuki KG, Fujiwara TK, Edidin M, Kusumi A. Dynamic recruitment of phospholipase $\mathrm{Cy}$ at transiently immobilized GPI-anchored receptor clusters induces $\mathrm{IP}_{3}-\mathrm{Ca}^{2+}$ signaling: single-molecule tracking study 2 . J Cell Biol 2007; 177: 731-42.

6 Uhlmann E, Peyman A, Breipohl G, Will DW. PNA: synthetic polyamide nucleic acids with unusual binding properties. Angew Chem Int Ed
1998; 37: 2796-823.

7 Shakeel S, Karim S, Ali A. Peptide nucleic acid (PNA) - a review. J Chem Technol Biotechnol 2006; 81: 892-9.

8 McNeer NA, Schleifman EB, Cuthbert A, Brehm M, Jackson A, Cheng $C$, et al. Systemic delivery of triplex-forming PNA and donor DNA by nanoparticles mediates site-specific genome editing of human hematopoietic cells in vivo. Gene Ther 2013; 20: 658-69.

9 Gaylord BS, Massie MR, Feinstein SC, Bazan GC. SNP detection using peptide nucleic acid probes and conjugated polymers: applications in neurodegenerative disease identification. Proc Natl Acad Sci U S A 2005; 102: 34-9.

10 Wang Q, Chen L, Long Y, Tian H, Wu J. Molecular beacons of xenonucleic acid for detecting nucleic acid. Theranostics 2013; 3: 395408.

11 Dueholm KL, Egholm M, Behrens C, Christensen L, Hansen HF, Vulpius $\mathrm{T}$, et al. Synthesis of peptide nucleic acid monomers containing the four natural nucleobases: thymine, cytosine, adenine, and guanine and their oligomerization. J Org Chem 1994; 59: 5767-73.

12 Christensen L, Fitzpatrick R, Gildea B, Petersen KH, Hansen HF, Koch $\mathrm{T}$, et al. Solid-phase synthesis of peptide nucleic acids. J Pept Sci 1995; 1: 175-83.

13 Thomson SA, Josey JA, Cadilla R, Gaul MD, Hassman CF, Luzzio MJ, et al. Fmoc mediated synthesis of peptide nucleic acids. Tetrahedron 1995; 51: 6179-94.

14 Ganesh KN, Nielsen PE. Peptide nucleic acids analogs and derivatives. Curr Org Chem 2000; 4: 931-43.

15 Egholm M, Christensen L, Deuholm KL, Buchardt O, Coull J, Nielsen $\mathrm{PE}$. Efficient $\mathrm{pH}$-independent sequence-specific DNA binding by pseudoisocytosine-containing bis-PNA. Nucleic Acids Res 1995; 23: 217-22.

16 Eldrup AB, Dahl O, Nielsen PE. A novel peptide nucleic acid monomer for recognition of thymine in triple-helix structures. J Am Chem Soc 1997; 119: 11116-7.

17 Eldrup AB, Nielsen BB, Haaima G, Rasmussen H, Kastrup JS, Christensen $\mathrm{C}$, et al. 1,8-Naphthyridin-2(1H)-ones. Novel bicyclic and tricyclic analogues of thymine in peptide nucleic acids (PNAs). Eur J Org Chem 2001; 2001: 1781-90.

18 Diederichsen U. Book review: peptide nucleic acids-methods and protocols. Edited by Nielsen PE. Chembiochem 2003; 4: 785-6.

19 Hyrup B, Nielsen PE. Peptide nucleic acids (PNA): synthesis, properties and potential applications. Bioorg Med Chem 1996; 4: 5-23.

20 Nielsen PE. Structural and biological properties of peptide nucleic acid (PNA). Pure Appl Chem 1998; 70: 105-10.

21 Demidov VV, Frank-Kamenetskii MD. Two sides of the coin: affinity and specificity of nucleic acid interactions. Trends Biochem Sci 2004; 29: $62-71$.

22 Betts L, Josey JA, Veal JM, Jordan SR. A nucleic acid triple helix formed by a peptide nucleic acid-DNA complex. Science 1995; 270 : 1838-41.

23 Egholm M, Buchardt O, Christensen L, Behrens C, Freier SM, Driver DA, et al. PNA hybridizes to complementary oligonucleotides obeying the Watson Crick hydrogen-bonding rules. Nature 1993; 365: 566-8.

24 Landgraf P, Rusu M, Sheridan R, Sewer A, lovino N, Aravin A, et al. A mammalian microRNA expression atlas based on small RNA library sequencing. Cell 2007; 129: 1401-14.

25 Krichevsky AM, Gabriely G. miR-21: a small multi-faceted RNA. J Cell Mol Med 2008; 13: 39-53.

26 Si ML, Zhu S, Wu H, Lu Z, Wu F, Mo YY, et al. miR-21-mediated tumor growth. Oncogene 2007; 26: 2799-803.

27 Bartel DP. MicroRNAs: genomics, biogenesis, mechanism, and 
function. Cell 2004; 116: 281-97.

28 Shivdasani RA. MicroRNAs: regulators of gene expression and cell differentiation. Blood 2006; 108: 3646-53.

29 Esquela-Kerscher A, Slack FJ. Oncomirs - microRNAs with a role in cancer. Nat Rev Cancer 2006; 6: 259-69.

30 Sassen S, Miska EA, Caldas C. MicroRNA-implications for cancer. Virchows Arch 2008; 452: 1-10.

31 Hayes J, Peruzzi PP, Lawler S. MicroRNAs in cancer: biomarkers, functions and therapy. Trends Mol Med 2014; 20: 460-9.

32 Dong $\mathrm{H}$, Lei J, Ju H, Zhi F, Wang H, Guo W, et al. Target-cell-specific delivery, imaging, and detection of intracellular microRNA with a multifunctional $\mathrm{SnO}_{2}$ nanoprobe. Angew Chem Int Ed 2012; 51: 4607-12.

33 Lee JS, Kin S, Na HK, Min DH. MicroRNA-responsive drug release system for selective fluorescence imaging and photodynamic therapy in vivo. Adv Healthc Mater 2016; 5: 2386-95.

34 Zhang MZ, Li C, Fang BY, Yao MH, Ren QQ, Zhang L, et al. High transfection efficiency of quantum dot-antisense oligonucleotide nanoparticles in cancer cells through dual-receptor synergistic targeting. Nanotechnology 2014; 25: 255102.

35 Zhang MZ, Yu Y, Yu RN, Wan M, Zhang RY, Zhao YD. Tracking the down-regulation of folate receptor- $\alpha$ in cancer cells through target specific delivery of quantum dots coupled with antisense oligonucleotide and targeted peptide. Small 2013; 24: 4183-93.

36 Stockwell BR. Frontiers in chemical genetics. Trends Biotech 2000; 18: 449-55.

37 Demidov VV. PNA comes of age: from infancy to maturity 'The PNA story is truly an amazing one'. Drug Discov Today 2002; 7: 153-5.

38 Nielsen PE. Peptide nucleic acids as therapeutic agents. Curr Opin Struct Biol 1999; 9: 353-7.

39 Hanvey JC, Peffer NJ, Bisi JE, Thomson SA, Cadilla R, Josey JA, et al. Antisense and antigene properties of peptide nucleic acids. Science 1992; 258: 1481-5.

40 Koller E, Gaarde WA, Monia BP. Elucidating cell signaling mechanisms using antisense technology. Trends Pharm Sci 2000; 21: 142-8.

41 Nielsen PE, Egholm M, Berg RH, Buchardt O. Sequence-selective recognition of DNA by strand displacement with a thymine-substituted polyamide. Science 1991; 254: 1497-500.

42 Suzuki T, Wu D, Schlachetzki F, Li JY, Boado RJ, Pardridge WM. Imaging endogenous gene expression in brain cancer in vivo with ${ }^{111}$ In-peptide nucleic acid antisense radiopharmaceuticals and brain drug-targeting technology. J Nucl Med 2004; 45: 1766-75.

43 Koppelhus U, Nielsen PE. Cellular delivery of peptide nucleic acid (PNA). Adv Drug Delivery Rev 2003; 55: 267-80.

44 Simmons CG, Pitts AE, Mayfield LD, Shay JW, Corey DR. Synthesis and membrane permeability of PNA-peptide conjugates. Bioorg Med Chem Lett 1997; 7: 3001-6.

45 Hamilton SE, Simmons CG, Kathiriya IS, Corey DR. Cellular delivery of peptide nucleic acids and inhibition of human telomerase. Chem Biol 1999; 6: 343-51.

46 Musilova K, Mraz M. MicroRNAs in B cell lymphomas: How a complex biology gets more complex. Leukemia 2015; 29; 1004-17.

47 Zhang GJ, Chua J, Chee RE, Agarwal A, Wong SM. Label-free direct detection of MiRNAs with silicon nanowire biosensors. Biosens Bioelectron 2009; 24: 2504-8.

48 Ficht S, Mattes A, Seitz O. Single-nucleotide-specific PNA-peptide ligation on synthetic and PCR DNA templates. J Am Chem Soc 2004; 126: 9970-81.

49 Ficht S, Dose C, Seitz O. As fast and selective as enzymatic ligations: unpaired nucleobases increase the selectivity of DNA-controlled native chemical PNA ligation. ChemBioChem 2005; 6: 2098-103.

50 Dose C, Ficht S, Seitz O. Reducing product inhibition in DNA-templatecontrolled ligation reactions. Angew Chem Int Ed 2006; 45: 536973.

51 Dose C, Seitz O. Single nucleotide specific detection of DNA by native chemical ligation of fluorescence labeled PNA-probes. Bioorg Med Chem 2008; 16: 65-77.

52 Wu J, Zou Y, Li C, Sicking W, Piantanida I, Yi T, et al. A molecular peptide beacon for the ratiometric sensing of nucleic acids. J Am Chem Soc 2012; 134: 1958-61.

53 Ostromohov N, Schwartz O, Bercovici M. Focused upon hybridization: rapid and high sensitivity detection of DNA using isotachophoresis and peptide nucleic acid probes. Anal Chem 2015; 87: 9459-66.

54 Hwang GT, Seo YJ, Kim BH. A highly discriminating quencher-free molecular beacon for probing DNA. J Am Chem Soc 2004; 126 : 6528-9.

55 Sandhya S, Chen W, Mulchandani A. Molecular beacons: a real-time polymerase chain reaction assay for detecting Escherichia coli from fresh produce and water. Anal Chim Acta 2008; 614: 208-12.

56 Takács T, Jeney C, Kovács L, Mózes J, Benczik M, Sebe A. Molecular beacon-based real-time PCR method for detection of 15 high-risk and 5 low-risk HPV types. J Virol Methods 2008; 149: 153-62.

57 Fang X, Li JJ, Perlette J, Tan W, Wang K. Peer reviewed: molecular beacons: novel fluorescent probes. Anal Chem 2000; 72: 747A753A.

58 Tan W, Wang K, Drake TJ. Molecular beacons. Curr Opin Chem Biol 2004; 8: 547-53.

59 Wang K, Tang Z, Yang CJ, Kim Y, Fang X, Li W, et al. Molecular engineering of DNA: molecular beacons. Angew Chem Int Ed 2009; 48: 856-70.

60 Nielsen PE. Peptide nucleic acid: a versatile tool in genetic diagnostics and molecular biology. Curr Opin Biotechnol 2001; 12 : 16-20.

61 Kam Y, Rubinstein A, Naik S, Djavsarov I, Halle D, Ariel I, et al. Detection of a long non-coding RNA (CCAT1) in living cells and human adenocarcinoma of colon tissues using FIT-PNA molecular beacons. Cancer Lett 2013; 352: 90-6.

62 Ortiz E, Estrada G, Lizardi PM. PNA molecular beacons for rapid detection of PCR amplicons. Mol Cell Probes 1998; 12: 219-26.

63 Tsourkas A, Behlke MA, Bao G. Structure-function relationships of shared-stem and conventional molecular beacons. Nucleic Acids Res 2002; 30: 4208-15.

64 Datta B, Armitage BA. Hybridization of PNA to structured DNA targets: quadruplex invasion and the overhang effect. J Am Chem Soc 2001; 123: 9612-9.

65 Singh RP, Oh BK, Koo KK, Jyong JY, Jeong S, Choi JW. Biosensor arrays for environmental pollutants detection. Biochip J 2008; 2: 223-34.

66 Brandt O. PNA microarrays for hybridisation of unlabelled DNA samples. Nucleic Acids Res 2003; 31: e119e.

67 Brandt O, Hoheisel JD. Peptide nucleic acids onmicroarrays and other biosensors. Trends Biotech 2004; 22: 617-22.

68 Perry-O'Keefe H, Yao XW, Coull JM, Fuchs M, Egholm M. Peptide nucleic acid pre-gel hybridization: an alternative to Southern hybridization. Proc Natl Acad Sci U S A 1996; 93: 14670-5.

69 Orum H, Nielsen PE, Jørgensen M, Larsson C, Stanley C, Koch T. Sequence-specific purification of nucleic acids by PNA-controlled hybrid selection. BioTechniques 1995; 19: 472-80. 